\title{
Scheduled Based Optimal Web Service Selection
}

\author{
Anuradha Kore' ${ }^{1}$ D. S. Bhosale ${ }^{2}$ \\ ${ }^{1,2}$ Computer Science \& Engineering, Shivaji University, Kolhapur, Maharashtra, India
}

\begin{abstract}
Effective service selection needs to fulfill both functional and non-functional requirements of users, non-functional requirements are concerned with the quality of service (QoS). QoS plays an important role in Web service recommendation, according to which similar services can be ranked and selected for users. The number of publicly available web services is steadily increasing on the internet. However, this proliferation makes it hard for a user to select a proper web service among a large amount of service candidates which provide similar functionalities. This research provides comprehensive information for selecting an optimal web service and predicting its behaviour in terms of RT. When user wish to use any service the system can provide recommendations, but only recommendation is not sufficient to improve QoS of the web service so that HMM \& scheduling is applied on the user requests to optimize the performance and reduce the response time; for more precise response time service provider allocate a service to the user before his request that is called pre-allocation. Overall aim of the research is to get well optimized web service which having great quality of service with very low response time.
\end{abstract}

Keywords: Response time, Recommendation, Pre-allocation, Service Scheduling, HMM

\section{Introduction}

In today ${ }^{\text {ee }}$ s world, the amount of web services is increases on internet, so that selection and recommendation of web service are becoming more important. Optimality of web service is depending on the performance of web service. Quality-of-Service (QoS) is widely employed to represent the non-functional performance of web services and has been considered as the key factor in service selection . Users usually get a list of web services from service brokers or search engines that meet the specific functional requirements. They need to identify the optimal one from the functionally equivalent candidates. Therefore it is difficult to select the best performing one, since service users usually have limited knowledge of their performance. The difficulty is selection of optimal web service.

Optimality of web service is depend on the performance of web service, performance is measures through quality of service i.e. QoS. Quality-of-Service (QoS) is usually defined as a set of nonfunctional properties, such as response time, throughput, etc. As the use of internet increases day by day everyone needs the response faster, but due to some high latency, heavy load problems, or poor performance of server users does not get that much of outcomes from the existing web service recommendation systems and does not get improved quality of services.

So in current state of art, selecting the best web service having extremely less response time is very important.

This research gives a novel approach for selecting the best web service by giving low response time and high QoS, with the help of scheduling, pattern mining and multiport server system. Scheduled based selection gives scheduling on user request, recommendation and pre-allocation of services and server cluster. There is one more issue regarding recommendation. To receive effective recommendations, users are required to supply their observed QoS values. Therefore the research proposes a simple yet effective privacy-preserving framework by applying data obfuscation techniques and anonymity.

\section{Motivation}

A QoS might be as useless as a service not providing the desired efficient results. It is impractical for every user to measure QoS performance of all the web service as there is large number of web services available. A web service is a software system designed to support interoperable machineto-machine interaction over a network. The number of publicly available web services is steadily increasing on the internet.

However, this proliferation makes it hard for a user to select a proper web service among a large amount of service candidates which provide similar functionalities. Quality of service is considered as the most important non-functional criterion for service selection. For internet services, the presence of low-performance servers, high latency or overall poor service quality can translate into lost sales, user frustration, and customers lost. This research provides comprehensive information for selecting an optimal web service and predicting it ${ }^{\text {ec }} \mathrm{s}$ behaviour in terms of RT. When user wish to use any service the system can provide recommendations, but only recommendation is not sufficient to improve QoS of the web service so that scheduling is applied on the user requests to optimize the performance and reduce the response time and for more precise response time service provider allocate a service to a user before his request that is called pre-allocation. Overall aim of the research is to get well optimized web service which having great quality of service with very low response time.

\section{Literature Review}

Waseem Ahmed propose a probabilistic model for predicting response time of web service and then selected an optimal web service at runtime from the list of functionally equivalent web services. To know the probabilistic insight of Web Services he had used Hidden Markove Model. Model 


\section{International Journal of Science and Research (IJSR) \\ ISSN (Online): 2319-7064 \\ Index Copernicus Value (2013): 6.14 | Impact Factor (2014): 5.611}

assumed that WS is deployed on a cluster of web servers and sometime the delay or crash during WS invocation is because the bad node in sever clustering responds to users ${ }^{\text {ee }}$ requests. With the help of HMM, he predicted the probabilistic behavior of these web servers and then selected the WS based on their probabilistic value [2]. According Tao has an efficient algorithm for selecting appropriate web services based on user"es provided weights. They have mapped web service composition with the Knapsack problem and then calculated the optimal path for executing useres requests [3]. Zibin Zheng, make the first attempt to cope with the privacy concerns for web service recommendation. Specifically, he proposes a simple yet effective privacy-preserving framework by applying data obfuscation techniques, and further develops two representative privacy-preserving QoS prediction approaches under this framework [4]. Rina Malik focuses on response time out of different QoS properties. In this approach the requests send by the user are scheduled on the basis of Earliest Deadline First. Then by combining the advantages of network coordinate based approach, simple regression, clustering approach and WSP approach, the response time between user and web services can be accurately predicted[5]. Priyanka D.Doltade doing the analysis of web usage of users based on the location, age along with other parameters which helps in improving the quality of web services in terms of providing faster web browsing to clients and based on their frequent usage of a particular website, ISP will provide buffering to those particular websites only [6]. Nikita R.Gurjar presents an innovative QoS-aware Web service recommendation approach .The basic idea is to predict Web services QoS values and recommends the best one for active users based on historical Web service QoS records. In order to better recommend Web services to users from amount of services with identical functions, he proposed a Web service recommendation approach based on collaborative filtering. In this paper, recommendation approach considered the correlation between QoS records and users physical locations by using IP addresses, which has achieved good prediction performance and makes the QoS prediction more confident for web service recommendation [7].

\section{Challenges Identified}

1)With the emergent number of Web services, it has become an urgent task to make operative selection from the large number of functionally-equivalent Web service candidates. There are number of different service provider and we have to effectively select the optimal one from web service candidates. In our approach the requests send by the user are scheduled on the basis of user and service priority scheduling.

2)It is impractical for each user to actively measure the QoS values due to the expensive overhead of invoking large number of service so that, to get more effective QoS and minimizing the response time recommendation and preallocation is used.

3)Collecting user ${ }^{\text {ec }}$ data for mining puts user privacy at risk; as a result privacy becomes challenge in developing a recommender system. So that privacy preserving is performed.

4)By combining the advantages of pattern mining, scheduling and the HMM model the overall response time required for the allocation of services is minimized.

\section{Problem Statements}

"To develop a system for selecting the web service having low response time and assuring good quality of service."

1)In proposed system user priority and service scheduling is performed for selecting better service from available services. Privacy preserving is also provided for the user security purpose.

2)Pattern mining gives the recommendation and preallocation. In recommendation by observing the user behavior next service is recommended as a suggestion, and the service is also already allocated so that response time will decreases.

3)Service provider contains web server cluster for load balancing and HMM model is used for giving probabilistic method of response time of services.

\section{Objectives}

The proposed work has following objectives,

1)Implement the system which gives user priority based scheduling

2)Implement service scheduling based on time and load balancing

3)Design and implement the system which gives recommendation and pre-allocation to users.

4) Implement a service provider which gives methodology for providing lowest response time to user. 


\section{International Journal of Science and Research (IJSR) \\ ISSN (Online): 2319-7064}

Index Copernicus Value (2013): 6.14 | Impact Factor (2014): 5.611

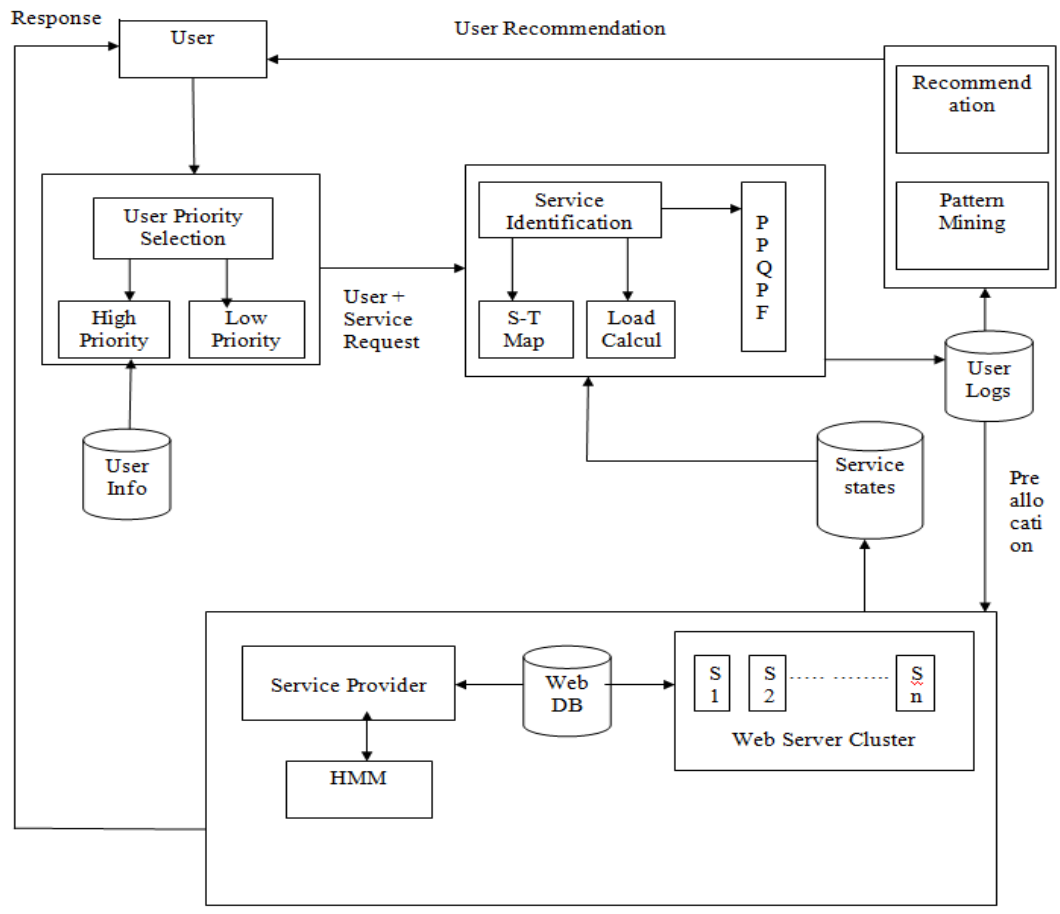

Figure 1: Architecture of Service Request Scheduling based Web Service Selection

Proposed work is based on selecting the optimal web service which is having improved quality of service. For implementing proposed work the system architecture is defined here which is having four different techniques that are used, first the user request is send to user priority identification module where scheduling is applied according to user authority and priority, after that service is identified at service time module, time and load scheduling is used for selecting better service out of all available. Whenever user wish to use the service his/her privacy issue arises so that privacy preserving is also performed by Privacy Preserving Quality of Service Prediction framework, then user logs are stored for applying pattern mining and by observing the patterns recommendation is send to user, at the same time pre-allocation of services is given to service provider after that service provider apply Hidden Markove Model on web services and give the optimized response time based web service with its future hidden states prediction and preallocation of services.

\subsection{Module 1: User Priority Scheduling}

User priority selection module make user request queue to process each user and provide priority to each user request based on user information. All user requests are stored into queue. Requests are assigned into different priority queues, these queues are served according to their priority from the highest to the lowest. High priority user treats as administrator users and provides fast response for their request. For low priority users response will base on the requested service. For this approach a novel method that is scheduling is used and scheduling is implemented with the help of user priority scheduling algorithm.

\subsection{Module 2: Service Request Scheduling}

In this approach service requests are scheduled according to time and load balancing algorithm. User service request response time is identified with the help of service identification module. This module keeps record of approximate execution time for each service. These records are arranged in format of Service- to-Time map. With the help of this map service scheduling is conducted by selecting first executing service with shortest response time.

In similar way service identification module keep records of current execution load on each service and update these records periodically. These load records are based on service state database operated by web service provider. Here user privacy is also secured with the help of Privacy Preserving Quality of Service Prediction framework (PPQPF) by data obfuscation and anonymity method.

\subsection{Module 3: Pattern mining}

This model gives the recommendation and pre-allocation of services to user. Nowadays weblog usage is tremendously increased. There is need of storing the log for further requirements and analysis. Web usage log store the activities of user from web sites. These activities can collect from web server or web browser. The user logs are stored, after that applying collaborative filtering and aprori algorithm, patterns is formed to predict the user behavior. This pre-allocation is base on future prediction of the recommended result selection probability.

\subsection{Module 4: Service provider}

In this approach user request is actually processed with HMM. It composed of different servers, providing list of 


\section{International Journal of Science and Research (IJSR) \\ ISSN (Online): 2319-7064 \\ Index Copernicus Value (2013): 6.14 | Impact Factor (2014): 5.611}

services to users, including web database and web server cluster. It provides direct response to user for his/her request. It also contains HMM as probabilistic methodology for response time predication. At the time of execution of a process it updates status of each service according to load on that service. These updates are managed in service states database which further will used by service identification module to perform scheduling task. Here for finding hidden states and hidden path the optimal web service selection algorithm is used.

\section{Conclusions}

A QoS might be as useless as a service not providing the desired efficient results. QoS plays an important role in Web service recommendation, according to which similar services can be ranked and selected for users. User gets optimal web service for his request. This web service selection interface is developing newly in proposed system. That is selection of services are done with the help of scheduling, pattern mining, probabilistic model for response time and pre-allocation of services is also provided. So the performance of web service selection will be optimized with assuring good quality of service. This paper gives the overall review of our proposed system.

\section{References}

[1] H. Zheng, J. Yang,W. Zhao, and A. Bouguettaya, ,„,QS Analysis for Web Service Compositions Based on Probabilistic QoS eee, in Service-Oriented Computing. Berlin, Germany: Springer-Verlag,2011, pp. 47-61.

[2] Тао YuÆ Kwei-Jay Lin "Service selection algorithms for Web services with end-to-end QoS constraints" ISeB (2005) 3: 103-126

[3] Waseem Ahmed "Response Time Based Optimal Web Service Selection”, IEEE Transaction On Parallel And Distributed Systems, vol. 26, NO. 2, February2015

[4] Jieming Zhu, Pinjia He, Zibin Zheng, "A PrivacyPreserving QoS Prediction Framework for Web Service Recommendation” 2015 IEEE International Conference on Web Services

[5] Reena MalikNisha Malik, "QSWSP: QoS Based Scheduled Web Service Positioning" International Journal of Scientific \& Engineering Research, Volume 4, Issue 11, November- 2013 ISSN 2229-5518

[6] Rashmi Saini "A Hybrid Algorithm for Load Balancing”, International Journal of Advanced Research in Computer Science and Software Engineering Volume 5, Issue 7, July 2015 ISSN: 2277 $128 \mathrm{X}$

[7] Priyanka D.Doltade "Analysis of WebLog Usage Mining For Intelligent Recommendation", International Journal of Integrated Computer Applications \& Research (Ijicar), vol-1

[8] Vidisha H. Zodape, Leena H. Patil "Literature Survey On Formation Of Association Rule Using Secure Mining", International journal of scientific \& technology research, volume 4, issue 01, january 2015 issn 2277-8616 222 ijstr@2015
[9] Nikita R.Gurjar1, Sandeep V.Rode2, "Personalized QoS-Aware Web Service Recommendation via Exploiting Location and Collaborative filtering", International journal of advanced research in computer science and software Engineering.vol 2015

[10] Jae-Hyun Cho, Han-Gyu Ko, and In-Young Ko, "Adaptive service selection according to the service density in multiple qos aspects", IEEE Transactions on Services Computing, DOI 10.1109/TSC.2015.2428251, 